\title{
Noncontact method of conducting elements "writing" on insulating Ge-Sb-Te matrix using a laser beam
}

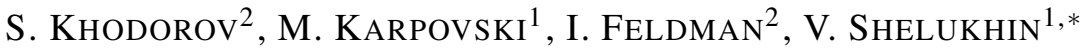 \\ ${ }^{1}$ Tel Aviv University, P.O. Box 39040, Tel Aviv 6997801, Israel \\ ${ }^{2}$ Weizmann Institute of Science, 234 Herzl St., P.O. Box 26, Rehovot 7610001, Israel
}

\begin{abstract}
Direct writing of low resistance wires on an amorphous $\mathrm{Ge}-\mathrm{Sb}-\mathrm{Te}$ matrix is reported. A $1342 \mathrm{~nm}$ continuous wave laser was used for local heating of the sample to form these wires. Mechanical contact was not needed for making the conducting elements. The properties of the samples were investigated down to $1.4 \mathrm{~K}$ and the laser conditions required for the writing of low resistance GST wires were found. The results are discussed with a view to possible applications, such as connectors and electrical wires made only via remote light exposure of the samples to make different resistors and non-linear elements.
\end{abstract}

Keywords: GST; laser; conductor; noncontact method

\section{Introduction}

Ge-Sb-Te compounds (GST) are exmples of a group of materials referred to as phase change materials. They have remained in the focus of scientific research for the last decade due to their outstanding physical properties [1-7]. Under temperature treatment, GST exhibits a reversible change from an amorphous phase to a crystalline hexagonal phase through intermediate crystalline phases (such as the rock salt phase), accompanied by a drastic drop (more than 4 decades) in resistance [8-10]. The changing of the crystallographic phases has been investigated using XRD analysis and was reported, for example, in the literature [11]. The process of crystallographic phases transformation can be stimulated not only by thermal treatment [1-5], but also by application of quasi-hydrostatic pressure [810,12 ] or by light which assists the formation of surface phonon-polaritons [13], or amorphization of the crystalline GST [14]. This family of chalcogenide glasses has a number of potential applications in the electronic industry [15-17]. The most important application is fabrication of memory cells.

\footnotetext{
*E-mail: Victorshe@post.tau.ac.il
}

We report on a new method of making conducting wires, based on crystallographic transformations of GST amorphous films under heating by continuous wave infrared radiation. These transformations are reflected in the electronic transport properties (such as resistivity) of the irradiated GST film areas. This method allowed us to make devices that consist of conducting wires with different properties using laser light only. In our experiments, we used a laser beam to heat the GST matrix locally in order to transform the desired areas into a conducting state while the whole GST matrix was kept unheated and non-conductive. Transport properties of the fabricated wires were investigated down to $1.4 \mathrm{~K}$.

\section{Experimental setup and mate- rial characterization}

We prepared our sample by RF magnetron sputtering in an argon plasma (base pressure $\sim 1.33 \times 10^{-5} \mathrm{~Pa}$ ) at an Ar pressure of 0.67 Pa using $\mathrm{GeSb}_{2} \mathrm{Te}_{4}$ targets (Kurt J. Lesker, 2 inch). The amorphous phase prior to local annealing by laser beam was confirmed by XRD analysis (Fig. 1a). The structure of the control sample ( $\mathrm{SiO}_{2}$ capped), which was prepared under the same sputtering 
conditions, after annealing (up to $282{ }^{\circ} \mathrm{C}$ ) in a furnace, was checked by XRD and was found in good agreement with the target Powder Diffraction File (PDF) database lines (Fig. 1b). This control experiment was made to confirm that we did not lose GST configuration during the sputtering process.
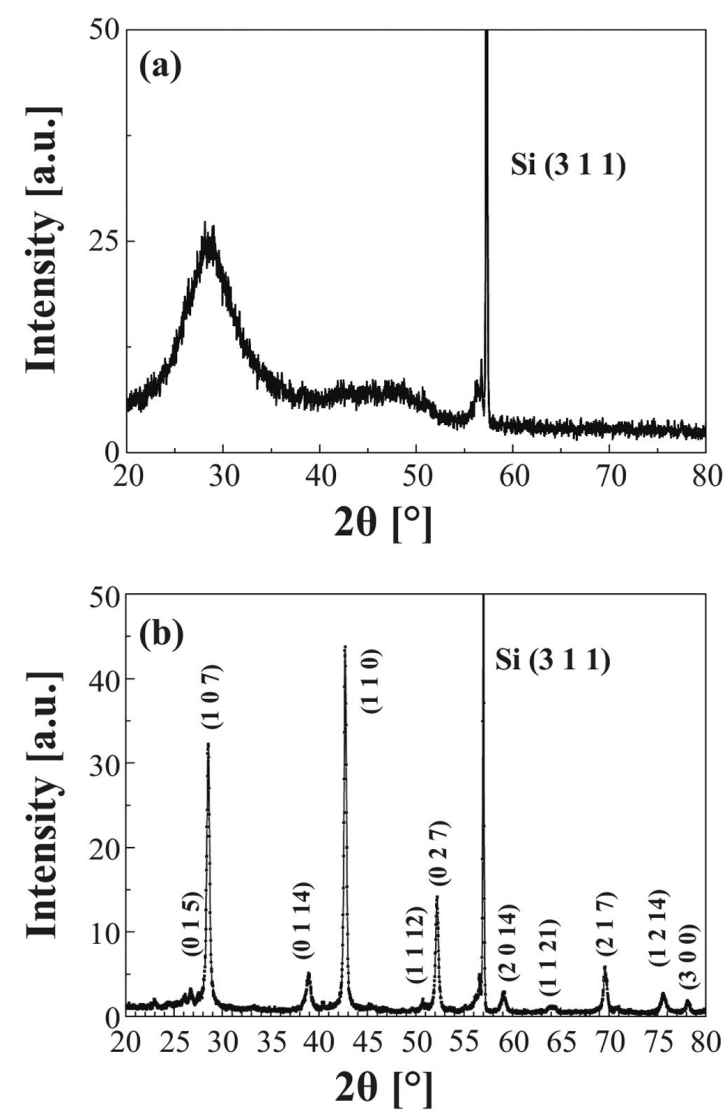

Fig. 1. XRD spectra of sputtered GST: (a) amorphous GST (sputtered on Si substrate together with the sample prior to "writing", which was made on GaAs; (b) control sample annealed up to $282{ }^{\circ} \mathrm{C}$ and PDF\# 97-008-5921 lines describing the GST target used in the experiments.

The amorphous films were subjected to the laser beam irradiation in the process of making conducting wires; this process is referred to in this article as laser beam writing (analogous to the e-beam writing process [18]). For this procedure, gold contacts were evaporated on the GaAs substrate and after that all the samples were covered by a $100 \mathrm{~nm}$ uniform and continuous layer of the sputtered GST, as was mentioned before.
The laser beam writing setup including an in situ measurement system is shown in Fig. 2. The laser beam writing system (Fig. 2a) consists of a $1342 \mathrm{~nm}$ continuous wave laser (1): MIL-H$1342-1.5 \mathrm{~W}$ with a focusing system, CCD camera (2): digital camera CCD/CMOS (the optics ensures magnification up to $300 \times$, at a working distance of $8 \mathrm{~cm}$, which allows obtaining in situ images during the experimental process) and the sample table (3) connected to the step-motor, which gives the possibility of slowing the sample movement during the experiment. Measurements of resistance were made using the electrical setup shown in Fig. 2b. The Keithley 2000 multimeter was directly connected to the sample which was thermally and electrically isolated from the table on which it was placed. The table was fixed on the motion stage (Precision Compact Linear Stage VP-25XL), which included the step-motor under control by a digital controller (Newport ESP301). The process was performed and controlled by a computer program written by us. The images and the electrical measurement data were automatically saved in the computer memory.

\section{Results and discussion}

More than ten samples were prepared, each with different wire configuration. Images of two of them at different stages of writing are shown in Fig. 3. We used the laser with the wavelength of $1342 \mathrm{~nm}$ and power which was varied between $0.8 \mathrm{~W}$ and $1.5 \mathrm{~W}$. The beam was focused to $60 \mu \mathrm{m}$; the average speed of sample motion with respect to the stationary laser was of $2.5 \mu \mathrm{m} / \mathrm{s}$ to $3 \mu \mathrm{m} / \mathrm{s}$. The sample with the size of $4 \mathrm{~mm} \times 4 \mathrm{~mm}$ and gold contacts to be bond to the chip pads, was placed on the chip.

It was found that the power required to make wires with minimal resistance was about $1.2 \mathrm{~W}$. In the case of power equal to $0.8 \mathrm{~W}$, the written lines are not seen, while if the power is $1.5 \mathrm{~W}$, the resistance of the wire is ten times greater than at the power of $1.2 \mathrm{~W}$. These facts are in good accord with calculations of temperature in affected region on the GST film, calculated using 


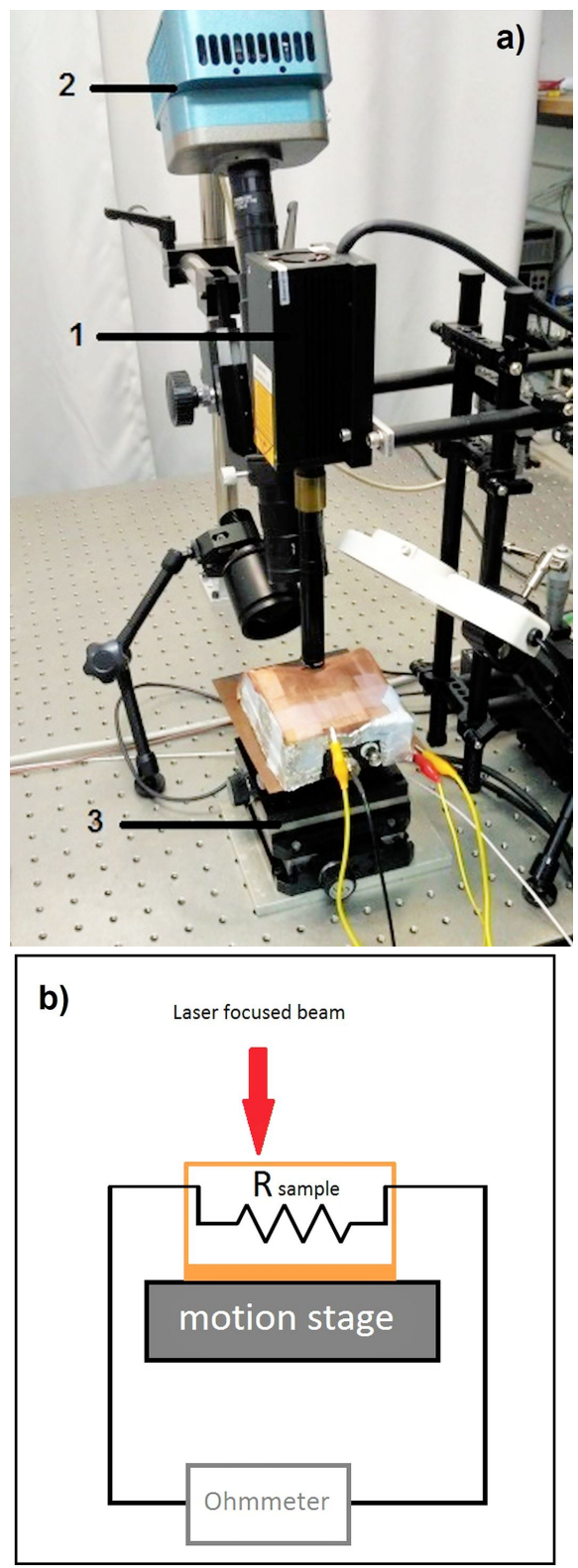

Fig. 2. (a) laser beam writing setup, 1 - laser, $2-C C D$ camera, 3 - sample table; (b) electrical system for in situ resistance measurement.

the formalism presented in the literature [19]. The temperature to which the substrate (GaAs) is heated is related to the energy accumulated by the substrate (the temperature of thin GST film is equal to the substrate temperature accordingly). For our substrate $(\mathrm{GaAs})$, the normalized laser power (p) has been calculated as:

$$
p=2[W(1-R)] / D
$$
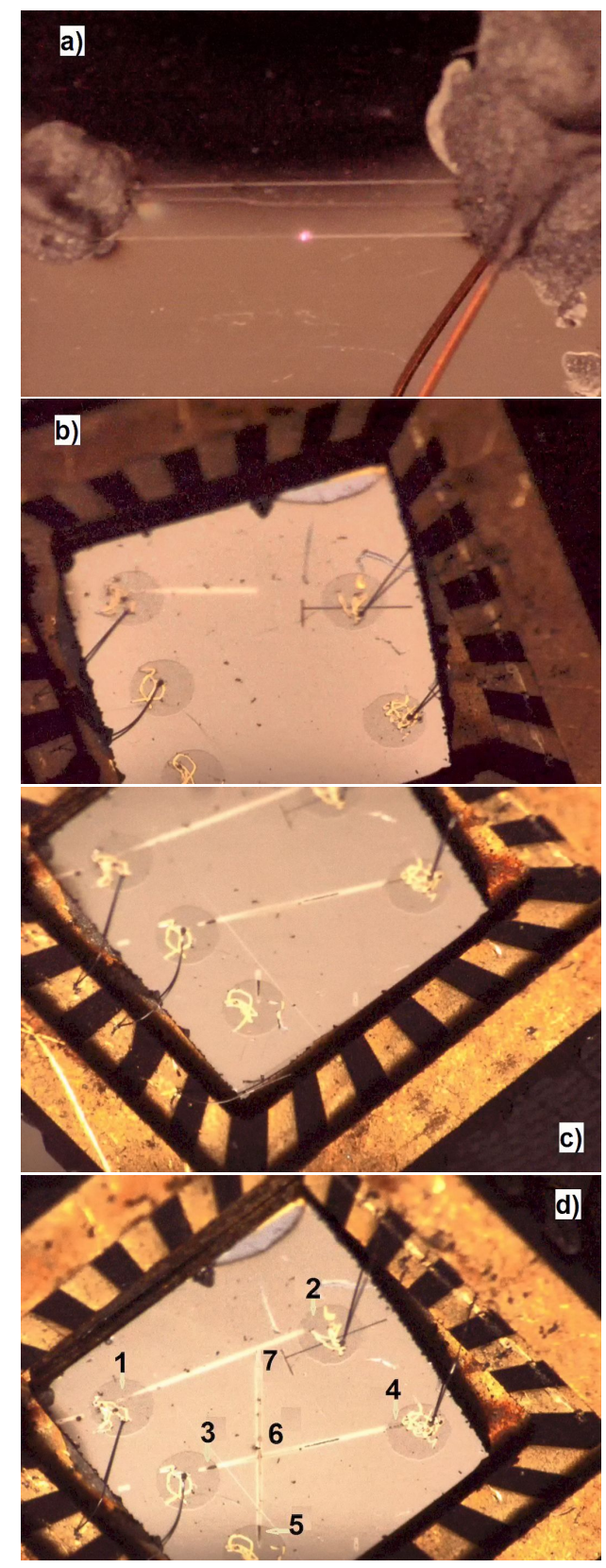

Fig. 3. Samples pictures: (a) first experiments with different laser beam powers; (b) to (d) writing of wires at different stages: (b) the first wire writing started; (c) two wires are made; (d) end of the writing, three wires are ready $(1 \rightarrow 2,3 \rightarrow 4$ and $5 \rightarrow 6 \rightarrow 7$ ).

where $\mathrm{W}$ is the laser power, $\mathrm{R}$ reflected part of the energy (reflectance), $\mathrm{D}$ the beam diameter, and $\mathrm{p}$ a power varying from $0.25 \mathrm{~W}$ to $0.8 \mathrm{~W}$ depending on temperature $\left(0.4 \mathrm{~W}\right.$ at $\sim 130{ }^{\circ} \mathrm{C}, 0.4 \mathrm{~W}$ to $1.2 \mathrm{~W}$ 
at $\sim 280{ }^{\circ} \mathrm{C}$ and $0.5 \mathrm{~W}$ to $1.5 \mathrm{~W}$ at $\sim 400{ }^{\circ} \mathrm{C}$ ). We assumed that only a part of the beam energy (about the half) is accumulated in the substrate. The reflected part can be calculated more accurately using the Fresnel equation for reflectance:

$$
R=\left[\left(n_{1}-n_{2}\right) /\left(n_{1}+n_{2}\right)\right]^{2}
$$

where $n_{1}$ and $n_{2}$ are the refractive indexes of two media. For the case of normal incidence (laser beam perpendicular to the sample), we received the reflectance of $36 \%$ to $56 \%$ (for air, to amorphous or GST crystal) and $0 \%$ to $11 \%$ (for GaAs and GST). The temperatures of the bottom substrate side were slightly different from room temperature. Using the formalism and estimations for GaAs from the literature [19], the maximum irradiance power density can be calculated as W/S (S is the irradiated area) and equals to $42 \mathrm{~kW} / \mathrm{cm}^{2}$ (taking into account light reflection, the value must be at least twice smaller than the incident irradiance [20]). A lasing power of $1.2 \mathrm{~W}$, which caused the substrate to heat to $\sim 280{ }^{\circ} \mathrm{C}$, was selected from the first experiments for the entire series. The samples were very sensitive to the exposure time, which is correlated directly with the average speed of the sample motion (Fig. 3): for $0.9 \mu \mathrm{m} / \mathrm{s}$ - the wire looks like the white line $(1 \rightarrow 2)$, for $1.05 \mu \mathrm{m} / \mathrm{s}$ - partly colored line $(3 \rightarrow 4)$, for $1 \mu \mathrm{m} / \mathrm{s}$ - the line looks like $(3 \rightarrow 4)$, but has more defects $(5 \rightarrow 6 \rightarrow 7)$.

We think that there are two reasons for this difference in the annealing results at longer exposure time: first, the different exposure times lead to different heating of the sample (change of the substrate backside temperatures [20]) and, as a result, to a difference in the annealing temperatures of the GST film; second, the difference in exposure time leads to an increase of the dwelling of GST at high temperature, and this greatly affects the GST relaxation, as it has been observed for samples regularly annealed in a furnace. The curves of the resistance measurements, which were recorded in situ during the wire's "writing", are shown in Fig. 4a. Despite the fact that the resistances at room temperature were close to each other and the resistivity varied from $2.0 \mathrm{~m} \Omega \cdot \mathrm{cm}$ to $2.4 \mathrm{~m} \Omega \cdot \mathrm{cm}$ at room temperature, the behavior of the wire $1 \rightarrow 2$ (long exposure) was completely different at low temperatures as is shown in Fig. 4b. The width of all wires was $60 \mu \mathrm{m}$ and the thickness was $100 \mathrm{~nm}$. The differently made wires were cooled down to $1.4 \mathrm{~K}$ to check their transport properties. The resistance of the $(1 \rightarrow 2)$ and $(3 \rightarrow 4)$ wires was measured by a lock-in-amplifier using a two-terminal connection scheme. These results are presented in Fig. $4 \mathrm{~b}$ and Fig. 4c. The resistance of the wire $(6 \rightarrow 7)$ (Fig. 4d), which exhibits the same behavior as the wire $3 \rightarrow 4$, was checked using a four-terminal connection scheme, which allowed to exclude the contacts influence. In this measurement we successfully checked the possibility to make a system consisting of more than one wire. Of course, this four-terminal system is independent of the contacts properties and their influence. The resistance was always measured between the first contact, where the writing started, and the second (last) point, where the writing was finished. The horizontal axis, $\mathrm{x}$, is the distance traveled by the laser beam from the first point. It means that the resistance presented on the graphs (Fig. 4) at any point $\mathrm{x}$ is a sum of the resistance of the written first contact point $\rightarrow \mathrm{x}$ wire and resistance of the remaining (not-traveled by the light) parts of the isolated matrix (area from $\mathrm{x}$ to the second contact point).

The wire $1 \rightarrow 2$ exhibits a very fast increase in resistance under cooling. Thus, two types of wires have been fabricated. The first type of wires is like that between contact 1 and contact 2 (long exposure), this wire exhibits a significant increase in resistance at low temperatures, which can be approximated by the Efros-Shklovskii variable range hopping law, (Fig. 5a), but does not exhibit a dependence of magnetic field (the data look like noise and are not presented in the picture to prevent camouflage of the presented curves), while the wires $3 \rightarrow 4$ and $6 \rightarrow 7$ exhibit small positive magnetoresistance (Fig. 5b), and the R(I) and V(I) curves looking essentially nonlinear (Fig. 5c and Fig. 5d).

The second type is the wires such as the wire between contact 3 and contact 4 (or $5 \rightarrow 6$, or $6 \rightarrow 7$ ) that are shown in the Fig. 3d. Their resistance increases very slowly, weakly depending 

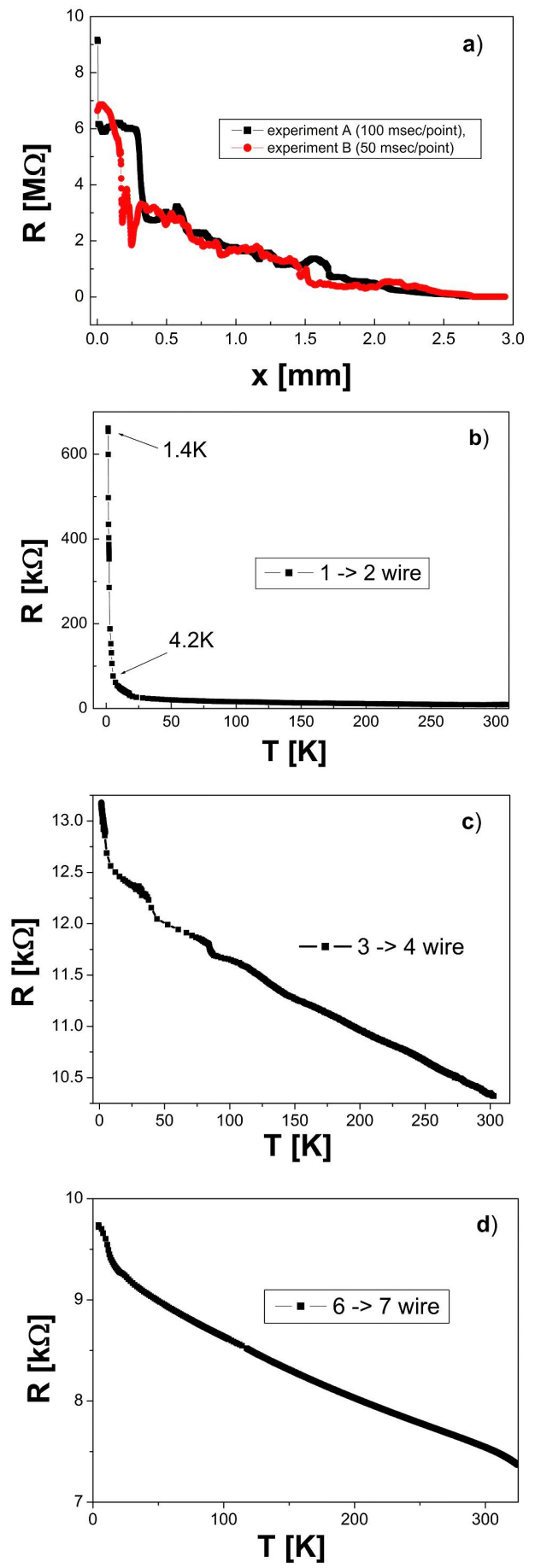

Fig. 4. (a) in situ measurement of $\mathrm{R}(\mathrm{x})$ for two different samples presented in Fig. 3a during writing, plotted as a function of temperature along different wires; (b) wire $1 \rightarrow 2$; (c) wire $3 \rightarrow 4$; (d) wire $6 \rightarrow 7$.
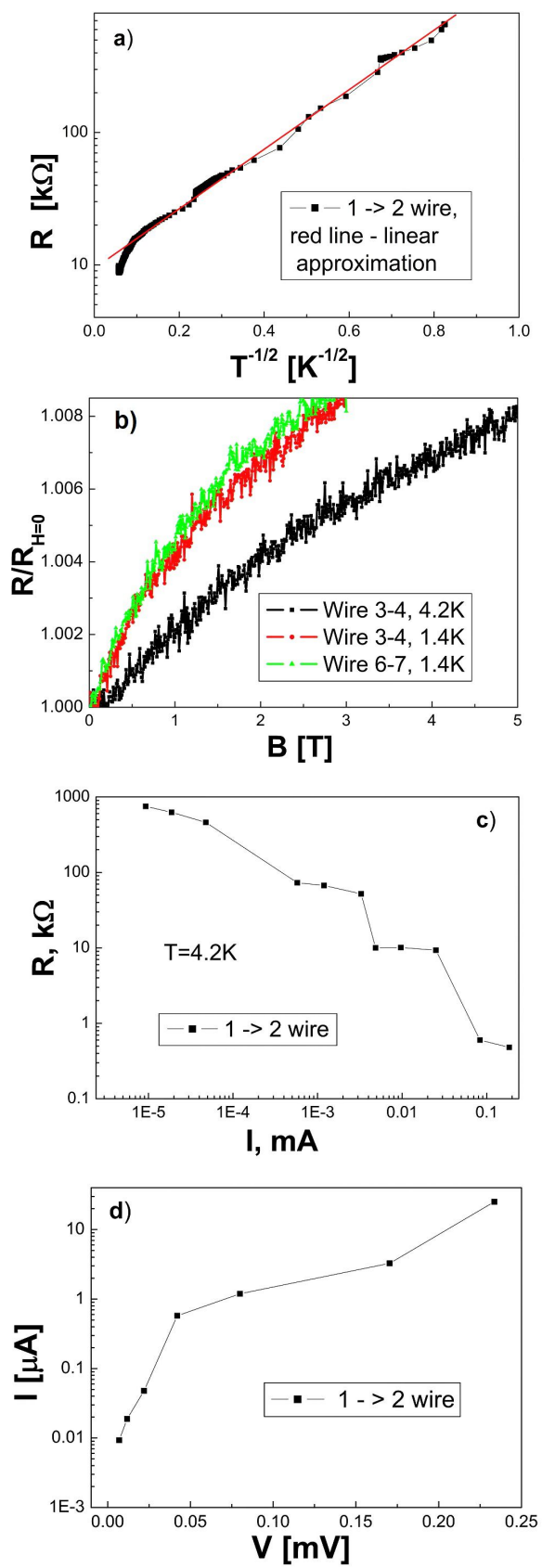

Fig. 5. (a) wire $1 \rightarrow 2$ resistance behavior under cooling (approximation by the Efros - Shklovskii law); (b) magnetoresistance, normalized on the value for zero field wires at $4.2 \mathrm{~K}$ and $1.4 \mathrm{~K}$, wire $6 \rightarrow 7$ at $1.4 \mathrm{~K}$, sheet resistance of $3 \rightarrow 4$ wire $\sim 260 \Omega / \square$ to $270 \Omega / \square, 6 \rightarrow 7$ wire $\sim 380 \Omega / \square$; (c) sample resistance R (I); (d) I-V characteristics measured at small currents. 
on the magnetic field (Fig. 5b). This behavior is the same as that of regularly annealed GST films which have been demonstrated in a series of experiments [1-5]. This type of wires can be used as connectors between any electric elements due to the very low resistance in comparison with amorphous isolating GST matrix around the wire. Therefore, enlarging the width of the wire leads to a proportional reduction of the wire resistance. It means that we can build resistors with different resistances using the same preparation conditions only making the wires of different width (we could write several overlapping wires, or change the laser beam diameter but the energy density of the laser beam and the thermal conditions during the process need to be kept the same).

We must emphasize that we have obtained the wires with completely different properties. The wire $3 \rightarrow 4$ looks like a GST sample annealed to a hexagonal phase (as it can be made by annealing in the furnace in the Ar atmosphere). We wish only to note, that the composition can be slightly different from the first composition $\left(\mathrm{Ge}_{0.95} \mathrm{Sb}_{2.01}\right) \mathrm{Te}_{4}$ since the heating is performed in air, but, as can be seen from the transport experiments, this has not not affected the properties of similar wires from $3 \rightarrow 4$ GST. The calculations gave the sheet resistance of this wire of $270 \Omega / \square$ at $1.4 \mathrm{~K}(210 \Omega / \square$ at room temperature). The calculations of resistivity yielded $2.1 \mathrm{~m} \Omega \cdot \mathrm{cm}$ at room temperature which is very close to the criterion of metal-insulating transition (the criterion is $1 \mathrm{~m} \Omega \cdot \mathrm{cm}$ to $2 \mathrm{~m} \Omega \cdot \mathrm{cm}$ as it was mentioned in the literature [1]. The wire $1 \rightarrow 2$ looks different. The resistance of wire $1 \rightarrow 2$ at room temperature is close to the resistance of samples like $3 \rightarrow 4$ (twice larger), while at low temperature the resistance of wire $1 \rightarrow 2$ is much higher than the resistance of wire $3 \rightarrow 4$. Furthermore, the weak dependence on the applied magnetic field for wire $3 \rightarrow 4$ corresponds to the transport measurement for this material [5] annealed by regular method in a furnace. The independence of the applied magnetic field for wire $1 \rightarrow 2$ instead of strong dependence, which is usually associated with the variablerange hopping regime, implies that the properties of this wire do not correspond to the regular GST behavior. Since this type of wires was made under the higher exposures we think that these samples were heated over $300{ }^{\circ} \mathrm{C}$ and that re-amorphization took place $[14,17]$. This process is accompanied by material desorption due to the high temperature heating produced in the air, and the energy barriers could be created on the grain boundaries and between the wires and contacts (the Schottky barriers). As a result, the resistance of wire $1 \rightarrow 2$ has a reactive part, while the active part is also enlarged. This type of wires cannot be used as connectors, but in devices where nonlinear I-V characteristics can be utilized: this could be a topic for a future study. We believe that the role of heat energy received from laser beam is to raise the temperature of the sample (including the substrate as well) locally to form a wire at the places where the laser beam is directed. As it was shown, the properties of the finished wires are strongly dependent on the parameters of the writing (laser parameters and exposure time). The surfaces phonon-polaritons have not formed in our case since we used the GaAs substrate (not quartz or any other polar crystal). It is important to mention that the substrate was not damaged by irradiation and it was absolutely clear after the GST layer removal.

As far as the samples quality and the usage conditions are concerned, the parameters of the wires have been stable for a half year, i.e., the period from the samples making to the present time. Therefore we have not checked the durability over longer periods yet, but we think that the wires could be covered by an insulating layer like $\mathrm{SiO}_{2}$ to prevent aging. Accordingly, temperatures above $300{ }^{\circ} \mathrm{C}$ are destructive for the wires parameters and these wires could be used only at lower temperatures; we think that their temperature should not exceed $200{ }^{\circ} \mathrm{C}$. In addition, we need to mention that these wires could be used in any low current electric schemes (the heating of the surrounding matrix by the heat due to the Joule-Lentz law does not lead to temperatures above $200{ }^{\circ} \mathrm{C}$ ). We have not registered any changes or significant heating using currents up to 0.1 A. Furthermore, the current flowing through the isolating areas is much lower and does not 
cause the sample heating. Even the I-V characteristics, presented in Fig. 5d, were measured at low currents to avoid points with the possibility of the samples being heated by the current.

An important issue is the quality and sharpness of the wire edges. The edges look sharp on the scale of the samples width and length. We not only observe the sharp edges by microscope, but suppose that the sharpness of the edges is in correspondence with the heat propagation. In other words, the distance between the wires and the unheated isolating matrix is not greater than $100 \mathrm{~nm}$ (GST thickness), it means about $0.16 \%$ of the wire width. In other words, the minimal distance between conducting elements should be about $400 \mathrm{~nm}$ to prevent significant leakage.

We hope that this method can be applied for making wires, for example, when an object can be made on a substrate covered by GST and the contacts to the object can be written after that. The second option is to cover a separate object and the contact pads with GST and to write on the GST after that. This option is the same as the process described in this paper consisting in making differently configured wires between existing contacts (or existing devices). This method could be very useful since we only need to fix the sample on the moving stage and focus the beam on, without physical contact, unlike in different bonder machines and other setups for making contacts.

\section{Conclusions}

1. The possibility to make conducting wires in an isolating matrix has been demonstrated using only a laser, which has regular characteristics.

2. The laser conditions for the manufacture of low resistance GST wires (without mechanical contact) were elaborated.

3. The prepared samples were characterized by transport experiments at room and low temperatures.

(a) The samples heated by laser below $280{ }^{\circ} \mathrm{C}$ had low resistance at room temperature (similar to dirty metals), which increased slowly during cooling (like in materials near the metal-insulator transition), and were characterized by a small positive magnetoresistance, as it is usually observed in metals.

(b) The overheated wires (above $300{ }^{\circ} \mathrm{C}$ ) demonstrated a strong increase of resistance during cooling as in non-metals, independently of the magnetic field and nonlinear $\mathrm{I}-\mathrm{V}$ characteristics.

4. The possibilities of applications, such as connectors and electrical wires made without mechanical contact with the samples, making different resistors and producing nonlinear elements, have been presented and discussed.

\section{Acknowledgements}

We would like to thank Prof. A. Palevski (Tel Aviv University) for helpful discussion on the subject of the paper. Our special thanks go to Prof. I. Lubomirski for the opportunity to use the equipment of his laboratory at the Weizmann Institute of Science. This research was supported in part by the PAZI Foundation under Grant No. 268/15.

\section{References}

[1] Siegrist T., Jost P., Volker H., Woda M., Merkelbach P., Schlockermann C., WutTIG M., Nat. Mater., 10 (2011), 202.

[2] Zhang W., Thiess A., Zalden P., Zeller R., DederichS P.H., RATY J.-Y., WutTIG M., Blugel S., Mazzarello R., Nat. Mater, 11 (2012), 952.

[3] Nukala P., Agarwal R., Qian X., Jang M.H., Dhara S., Kumar K., Johnson A.T.C., Li J., AgARWAL R., Nano Lett., 14 (2014), 2201.

[4] Siegert K.S., Lange F.R.L., SitTner E.R., Volker H., Schlockermann C., Siegirst T., Wuttig M., Rep. Prog. Phys., 78 (2015), 013001.

[5] Brezenay N.P., Volker H., Palevski A., MazZarello R., Kapitulnik A., Wuttig M., Phys. Rev. B, 86 (2012), 205302.

[6] Zalden P., Siegert K.S., Rols S., Fischer H.E., Schlich F., Hu T., Wuttig M., Chem. Meter, 26 (2014), 2307.

[7] Lencer D., SAlinga M., Grabowski B., Hickel T., Neugebauer J., Wuttig M., Nat. Mater, 7 (2008), 972.

[8] Xu M., Cheng Y.Q., Wang L., Sheng H.W., Meng Y., Yang W.G., Han X.D., Ma E., Proc. Natl. Aacad. Sci.USA, 109 (2012), E1055. 
[9] Sun Z., Zhou J., Pan Y., Song Z., MaO H.-K., Ahuja R., Proc. Natl. Acad. Sci. USA, 108 (2011), 10410.

[10] Xu M., Zhang W., Mazzarello R., Wuttig M., Adv. Sci., 2 (2015), 1500117.

[11] Kalkan B., Sen S., Cho J.-Y., Joo Y.-C., Clark S.M., Appl. Phys. Lett., 101 (2012), 151906.

[12] Greenberg E., Hen B., Layek S., Pozin I., FriedMAN R., Shelukhin V., Rosenberg Y., KARPOVSKI M., PASTernak M.P., Sterer E., DAGan Y., Rozenberg G.KH., Palevski A., Phys. Rev. B, 95 (2017), 064514.

[13] Li P., YAnG X., Mass T.W.W., Hanss J., Lewin M., Michel A.-K.U., Wuttig M., Taubner T., Nat. Mater, 15 (2016), 870.

[14] Siegel J., Gawelda W., Puerto D., DorRonsoro C., Solis J., Afonso C.N., SAnde DE J.C.G., Bez R., Pirovano A., Wiemer C., J. Appl. Phys., 103 (2008), 023516.
[15] Loke D., LeE T.H., Wang W.J., Shi L.P., ZhaO Y.C., Chong T.C., Elliott S.R., Science, 336 (2012), 1566.

[16] Rios C., Stegmaier M., Hosseini P., Wang D., Scherer T., Wright C.D., Bhaskaran H., PerNICE W.H.P., Nat. Photonics, 9 (2015), 725.

[17] Wuttig M., Yamada N., Nat. Mater, 6 (2007), 824.

[18] Tseng A.A., Chen K., Chen C.D., Ma K.J., IEEE Trans. Electron. Packag. Manuf., 26 (2), (2003), 141.

[19] Pernice W.H.P., Bhaskaran H., Appl. Phys. Lett., 101 (2012), 171101.

[20] Nissim Y.I., Lietoila A., Gold R.B., GibBons J.F., J. Appl. Phys., 51 (1980), 274.

Received 2017-09-18

Accepted 2018-01-28 